\title{
Network Reconfiguration with Placement of DGs to Minimize the Power Loss in RDSs using CABC Algorithm
}

\author{
N. Mohandas, $\mathrm{PhD}$ \\ Lecturer \\ Department of Electrical and Electronics Engineering \\ Government Polytechnic College \\ Srirangam
}

\begin{abstract}
The network reconfiguration is reshaping of the network system with supporting of sectionals/tie switches so as to reduce the power loss and to improve the voltage profile of the system. This paper presents the reconfiguration of a network system with location of distributed generation (DG) is to reduce power loss and in order to improve the voltage stability of the radial distribution systems (RDS). In this approach, the objective function is formulated based on the various technical issues such as power losses, thermal limit, voltage profile and stability of the system. The network reconfiguration problem is a nonlinear optimization problem; a chaotic artificial bee colony (CABC) algorithm is implemented to find the optimal solution of this approach. It is one of the enhanced versions of artificial bee colony algorithm. Two different cases are considered of this approach such as (i) only reconfiguration and (ii) reconfiguration with DGs. The efficiency of the proposed algorithm is validated by testing it on 33-node and 69-node radial distribution systems. The simulation results of this proposed approach are compared with other methods available in the earlier report.
\end{abstract}

\section{Keywords}

Chaotic artificial bee colony, network reconfiguration, distributed generation, distribution system, power loss, voltage stability.

\section{INTRODUCTION}

The radial distribution system is an interconnection between the customer and transmission network. The various load models are considered in the distribution systems such as residential, industrial and commercial. The loading pattern of these load models varies with wide range according to the peak hours. During peak load conditions, the voltage magnitude of some buses exceeds the voltage value of permissible limit. It affects the voltage stability of the distribution system. The network reconfiguration of the distribution system is used to reduce the power loss and to improve the voltage profile and stability of the system.

Network reconfiguration is altering the topological structure of distribution feeders by changing the status of sectionalizing switches, and is usually done for real power loss reduction and load balancing in the distribution system [1]. The authors' presents a maximum load ability index based optimal network reconfiguration of radial distribution systems [2]. The quadratic-loss allocation scheme such as branch current flow and ensures that every consumer has allocated losses at branches for which current it contributes [3]. The load balance index based approach the optimal network reconfiguration of the distribution system using a Plant Growth Simulation algorithm is reported in [4]. The particle swarm optimization (PSO) algorithm based approach to distribution feeder reconfiguration with presence of DG units [5].The refined genetic algorithm (RGA) based reconfiguration of the distribution network in order to minimize the power loss in the system is reported in [6].

The harmony search algorithm (HSA) based feeder reconfiguration of the distribution system with the objective of improving the voltage profile and minimizing real power loss of the system. The different scenarios such as reconfiguration and reconfiguration with DG are considered and satisfied the constraints of the distribution system are discussed in $[7,8]$. The two stage procedure such as power loss sensitivity information and branch exchanges are in literature [9] and which has used to improve the solution of network reconfiguration. In [10], the voltage stability index (VSI) based reconfiguration of radial distribution systems using a fuzzy genetic algorithm. The capacitor is mainly used for reactive power compensation in radial distribution system. The simultaneously process of network reconfiguration and location of capacitors is intended to reduce the real power loss of distribution systems using different soft computing techniques such as ant colony search (ACS) and improved binary particle swarm optimization (IBPSO) algorithms in literatures $[11,12]$.

DG generally refers to small-scale electric power generators that produce electricity at a site nearby customers or that are coupled to an electrical distribution system [13]. The fuzzygenetic algorithm (GA) based optimal placement of fixed and switched capacitors are used to improve the voltage profile of radial distribution system [14]. The various technical issues such as power loss, line flow limit and voltage profile are considered and which are related to the impact indices are used to obtain the optimal location and sizing of single and multiple DG units in the distribution system [15 - 17]. The new voltage stability index is presented in the system and which is used to identify the most sensitive node of the radial distribution system [18]. The power flow algorithm method exploits the radial nature of the network and uses forward and backward propagation to determine branch currents and node voltages [19]. A simple and efficient load flow technique for solving radial distribution system is developed in [20].Artificial Bee Colony (ABC) algorithm is an optimization algorithm based on the intelligent foraging behaviour of honey bee swarm. The ABC algorithm employs a different selection of operators such as greedy selection, probabilistic selection and random selection of the food 
sources. The ABC algorithm is a successful tool for solving large scale optimization problems with constraints in order to feasible regions of entire search space $[21,22]$. The ABC algorithm is used for solving a large set of numerical test functions. The optimal results of numerical test function using $\mathrm{ABC}$ algorithm is improved when compared to the results of other soft computing techniques such as genetic algorithm, particle swarm optimization algorithm and differential evolution algorithm [23]. The modification rate (MR) is one of the additional control parameter and which is used to modify the ABC algorithm is discussed in [24]. To improve the local intensification capability of the $\mathrm{ABC}$ algorithm has introduced a self adaptive strategy used for generating neighboring food sources based on the insert and swap operators is developed to enable the discrete $\mathrm{ABC}$ algorithm [25]. The local search performance of particle swarm optimization (PSO) algorithm is improved by incorporating chaos theory is discussed in [26]. The weighting factors are selected to the objective function is important to the objective of the optimization problem and is not important to the relative magnitudes of the function values [27].

This paper investigates the planning issues for the main concern of network reconfiguration and DG placement problems in the radial distribution systems using CABC algorithm. The local search capability of an optimization problem is improved using a chaos theory and is combined with $\mathrm{ABC}$ algorithm called $\mathrm{CABC}$. The multi objective performance index (MOPI) is formed by using the various impact indices reported in [17]. To assess the proposed approach up to two cases and which can affect the planning issues in the distribution system has been investigated. Simulation results proved the potential of this algorithm for identifying the optimal network reconfiguration and placement and sizing of DGs in the radial distribution systems.

This article is organized as follows. Section 2 discusses with the various impact indices, which are related to the technical issues of the radial distribution system. Section 3 extends the impact indices which are associated to form the MOPI and is used to propose the planning issues of the distribution system. The important power flow model is given in section 4 . The chaotic artificial bee colony algorithm is completely described in Section 5. Section 6 gives the implementation details and the results. Section 7 presents important conclusions.

\section{IMPACT INDICES}

First of all, the two optimal cases are considered of this approach and to study the superiority of the proposed method.

Case I: The system is optimized with the network reconfigured condition.

Case II: The system is optimized with simultaneously the process of network reconfigured, location and sizing of DGs.

In this approach, the indices are considered with various technical issues and which are associated to form the MOPI. These impact indices are defined as follows,

\subsection{Real and Reactive Power Loss Index (ILP and ILQ)}

The reasonable reduction of real and reactive power losses in the distribution system which results of system operates in maximum benefit. These power loss indices are define as [17],

$$
\begin{gathered}
I L P=\frac{\left\lfloor P_{\text {optimalca. }}\right\rfloor}{\left[P_{L}\right\rfloor} \\
I L Q=\frac{\left\lfloor Q_{\text {optimalca. }}\right\rfloor}{\left[Q_{L}\right\rfloor}
\end{gathered}
$$

where, $P_{\text {optimalcase }}$ and $Q_{\text {optimalcase }}$ are the total real and reactive power loss of the distribution system according to the case condition. $P_{L}$ and $Q_{L}$ are the total real and reactive power loss of the distribution system for the base case condition.

\subsection{Voltage Stability Index (VSI)}

The VSI gives the information about whether the system operates in secure or insecure condition. It is taken as the important factor and which is a variation of this value indicating that the system voltage stability due to the optimal case. The VSI can be defined as [18],

$$
\begin{aligned}
V S I(m 2)= & |V(m 1)|^{4}-4.0\{P(m 2) x(j j)-Q(m 2) r(j j)\}^{2} \\
& -4.0\{P(m 2) r(j j)+Q(m 2) x(j j)\}|V(m 1)|^{2}
\end{aligned}
$$

where,

$\begin{array}{ll}\mathrm{NN} & \text { - total number of nodes } \\ j j & \text { - branch number } \\ \mathrm{VSI}(\mathrm{m} 2) & \text { - voltage stability index of } \\ \text { node } \mathrm{m} 2,(\mathrm{~m} 2=2,3 \mathrm{NN}) \quad r(j j) & - \text { resistance } \\ \text { of branch } j j & \\ x(j j) & \text { - reactance of branch } j j \\ \mathrm{~V}(\mathrm{~m} 1) & \text { - voltage at node } \mathrm{m} 1 \\ \mathrm{~V}(\mathrm{~m} 2) & \text { - voltage at node } \mathrm{m} 2 \\ \mathrm{P}(\mathrm{m} 2) & \text { - reactive power load fed through node m2 } \\ \mathrm{Q}(\mathrm{m} 2) & \end{array}$

When the system operates in secure condition the determined value of VSI is greater than zero, otherwise insecure occurs.

\subsection{Current Capacity Index (CC)}

During the optimal case condition, the system significantly changes the current flow in various branches of the network. According to these flows is within limit means which represents that the feeder is kept away from overloading. The value of this index is less than unity indicates that the permissible limits of current flow and to avoid overloading of the feeder. 


$$
C C=\max _{i=1}^{N B}\left(\frac{\left|\bar{I}_{i j}\right|}{\left|\bar{I}_{\max i j}\right|}\right)
$$

where,

$$
\begin{array}{ll}
\bar{I}_{i j} & - \text { current flows of branch } i \text { and } j \\
\bar{I}_{\max i j} & -\begin{array}{l}
\text { maximum current carrying capacity of } \\
\text { branch } i \text { and } j
\end{array} \\
& \text { NB }
\end{array}
$$

\subsection{Voltage Profile Index (IVD)}

The voltage profile related to the IVD index can be defined as follows [17],

$$
I V D=\max _{i=2}^{N N}\left(\frac{\left|\bar{V}_{\text {nominal }}\right|-\left|\bar{V}_{i}\right|}{\left|\bar{V}_{\text {nominal }}\right|}\right)
$$

where,

$$
V_{\text {nomin } a l}=1.00 \text { p.u. }
$$

$\mathrm{NN}$ - number of nodes.

The value of this index is normally small and which is represented that the voltage limit is properly maintained and voltage profile of the system is greatly improved.

\section{PROBLEM FORMULATION}

The MOPI is a several impact indices and it is used to propose the network reconfiguration with optimal placement and sizing of DG units in order to reduce the power loss of the radial distribution systems. The major value of VSI is to improve the voltage stability of the distribution system and the minor values of ILP, ILQ, CC and IVD are to minimize the power losses, current flows are within limit and to improve the voltage profile of the distribution system respectively. For superior performance of the distribution system, the separate indices (except VSI) are normalized between zero and one. The separate indices are combined with help of weighting factor to form the MOPI.

The chaotic artificial bee colony algorithm based multi objective performance index (MOPI) is given by,

$M O P I=\omega_{1} \cdot I L P+\omega_{2} . I L Q+\omega_{3} \cdot\left(\frac{1}{V S I}\right)+\omega_{4} \cdot C C+\omega_{5} . I V D^{(6)}$

where, $\sum_{P=1}^{5} \omega_{P}=1.0 \Lambda \omega_{P} \in[0,1]$

The weighted sum method is used to design the multi objective optimization problem. The various objective functions are to multiply the weighting factors and summing the results to form the aggregated objective function. The value of weighting factors is decided according to the network designer. In this approach, the first part of the objective function is considered as a real power loss index and is receiving a weight factor is 0.35 . The second part of the objective function is the reactive power loss index and has received 0.15 as a weight factor. The VSI receives 0.10 , gives the information of voltage stability of the radial distribution system. The $\mathrm{CC}$ index receives 0.25 gives the details about line upgrading. The latter IVD index receives a weight factor of 0.15 .

The network reconfiguration is the process of changing the structure of the distribution system with help of sectionals/tie switches. For changing this way of structure is to maintain the radial structure of the network and should be satisfied the constraints. These constraints are discussed as follows.

\subsection{Power - Conservation Limits}

The algebraic sum of all receiving and sending powers with line losses over the entire radial distribution system and power produced from the DG unit should be equal to zero.

$$
\begin{aligned}
& P_{S S}=\sum_{i=2}^{N B} P_{D}(i)+\sum_{j=1}^{N L} P_{\text {loss }}(j)-\sum_{k=1}^{N D G} P_{D G}(k) \\
& Q_{S S}=\sum_{i=2}^{N B} Q_{D}(i)+\sum_{j=1}^{N L} Q_{\text {loss }}(j)
\end{aligned}
$$

where,

$P_{S S}$ - real power conservation by the system (MW)

$P_{D} \quad$ - total system real power demand (MW)

$P_{\text {loss }}$ - total system real power loss (MW)

$P_{D G}$ - total real power generated by DG (MW)

$Q_{S S}$ - reactive power conservation by the

system (MVAr)

$Q_{D}$ - total system reactive power

demand (MVAr)

$Q_{\text {loss }} \quad$ - total system reactive power loss (MVAr)

$N D G \quad$ - number of DG.

The power conservation limits of the network reconfiguration case, the above equation (7) in absents of $\mathrm{P}_{\mathrm{DG}}$.

\subsection{DG Real Power Generation Limits}

The real power generated by each DG $\left(P_{D G}\right)$ is limited by its boundary limits as,

$$
P_{D G}^{\min } \leq P_{D G} \leq P_{D G}^{\max }
$$

\subsection{Voltage Profile Limits}

The voltage magnitude at each node of the radial distribution system is defined as,

$V_{i}^{\min } \leq V_{i} \leq V_{i}^{\max }$

$0.90 \leq V_{i} \leq 1.00$ (Both test systems).

where, $\begin{array}{ll}V_{i} & \text { - voltage magnitude of node } i \\ V_{\min }, V_{\max }, & \text { - minimum and maximum node voltage } \\ \text { magnitude limits. } & \end{array}$

The voltage at each node of the radial distribution system should be maintained within the permissible limits 


\subsection{Line Thermal Limits}

The current flows in the branches should not go beyond the thermal capacity of the line.

$I_{(i, j)} \leq I_{(i, j)}^{\max }$

where,

$I_{(i, j)}, I_{(i, j)}^{\max } \quad$ - current magnitude and maximum current limit of branch $(i, j)$.After connecting of tie lines, the committed nodes are to maintain the radial nature of the system and to satisfy the constraints, the network reconfiguration allows for next generation of population. Otherwise, rejects the network reconfiguration in the next generation.

\section{POWER FLOW ALGORITHM}

The Power flow plays an important role of network reconfiguration of distribution systems. It is assumed that the three-phase radial distribution networks are balanced and can be represented by their equivalent single line diagrams [20].
Figure 1 shows a single - line diagram of a radial distribution network. The branch number sending-end and receiving-end node of this feeder are given in Table 1.

The load current at any node $i$, is given as

$$
I L(i)=\frac{P L(i)-j Q L(i)}{V_{(i)}^{*}} \quad i=2,3, \ldots ., \mathrm{NN}
$$

where,

$I L(i) \quad-$ load current of node $i$

$P L(i), Q L(i) \quad$ - real and reactive power load at $i^{\text {th }}$ node.

The charging current at node $i$ is

$$
I C(i)=y_{0}(i) V(i) \quad i=2,3, \ldots ., \mathrm{NN}
$$

where,

IC $(i)$ - charging current at node $i$

charging admittance

at

node $i$

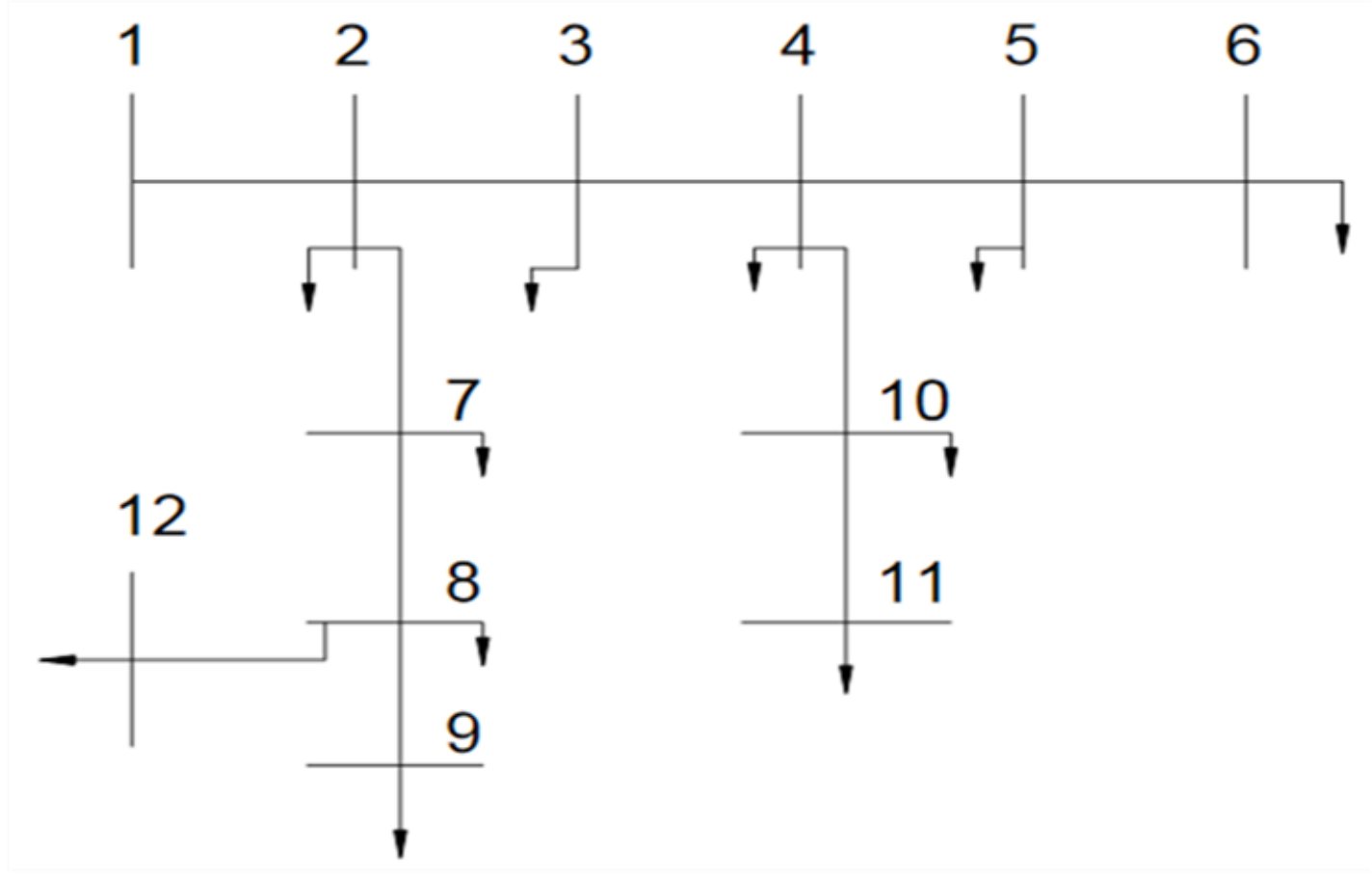

Fig. l. Single line diagram of radial distribution network 
Table 1 Branch number, sending end node, receiving end node, and nodes beyond different branches

\begin{tabular}{|c|c|c|c|c|}
\hline $\begin{array}{c}\text { Branch Number } \\
(j j)\end{array}$ & $\begin{array}{c}\text { Sending end } \\
m I=I S(j j)\end{array}$ & $\begin{array}{c}\text { Receiving end } \\
m 2=I R(j j)\end{array}$ & $\begin{array}{c}\text { Nodes beyond } \\
\text { branch } \ddot{j j}\end{array}$ & $\begin{array}{c}\text { Total number } \\
\text { of nodes } N(j j) \\
\text { beyond branch } j j\end{array}$ \\
\hline 1 & 1 & 2 & $2,3,4,5,6,7,8,9,10,11,12$ & 11 \\
\hline 2 & 2 & 3 & $3,4,5,6,10,11$ & 6 \\
\hline 3 & 3 & 4 & $4,5,6,10,11$ & 5 \\
\hline 4 & 4 & 5 & 5,6 & 2 \\
\hline 5 & 5 & 6 & 6 & 1 \\
\hline 6 & 2 & 7 & $7,8,9,12$ & 4 \\
\hline 7 & 7 & 8 & $8,9,12$ & 3 \\
\hline 8 & 8 & 9 & 9 & 1 \\
\hline 9 & 4 & 10 & 10,11 & 2 \\
\hline 10 & 10 & 11 & 11 & 1 \\
\hline 11 & 8 & 12 & 12 & 1 \\
\hline
\end{tabular}

Current through any one of the branch is equal to the sum of the load currents of all the nodes beyond this branch plus the sum of the charging currents of all the nodes beyond this branch. The branch current is given as,

$I(j j)=\sum_{i=1}^{N(j j)} I L(j j, i)+\sum_{i=1}^{N(j j)} I C(j j, i) \quad j j=1,2, \ldots ., \mathrm{NB}$

where,

$$
\mathrm{N}(j j) \quad \text { - total number of nodes beyond branch } j j \text {. }
$$

For instance, the current through branch 6 is equal to the sum of the load currents of all the nodes beyond branch 6 plus the sum of the charging currents of all the nodes beyond branch 6 , which represents that,

$$
\begin{aligned}
I(6)= & I L(7)+I L(8)+I L(9)+I L(12)+I C(7)+ \\
& I L(8)+I L(9)+I L(12)
\end{aligned}
$$

Consequently, there is known the nodes beyond all the branches, it is able to evaluate all the branch currents in the system. Load currents and charging currents are computed iteratively.

The real and reactive power of branch $j j$ are given by,

$$
L P(j j)=|I(j j)|^{2} R(j j)
$$

$L Q(j j)=|I(j j)|^{2} X(j j)$

\section{ARTIFICIAL BEE COLONY (ABC) ALGORITHM}

The artificial bee colony (ABC) algorithm is built up based on intelligent performance of honey bee swarm [21]. The searching strategy of food source in this algorithm the following three steps such as,

- $\quad$ Employed bees

- Onlooker bees

- $\quad$ Scout bees
Employed bees: In the first step of this algorithm, to search for new food sources in the dancing area having more nectar within the neighbourhood of the food source.

Onlooker bees: It is waiting for the dance area in the hive, and which is share the information about employed bees. To search for new food source of these bees based on probability and related to the shared information.

Scout bees: If a specific food source solution cannot be improved with maximum number of trials, it will be indicate that the solution is abandoned. Scout bees used to replace the abandoned solution of food source.

Additionally, three control parameters, use of this algorithm such as population size, maximum cycle number and limit.

\subsection{Chaotic local search}

The chaos theory is used to improve the local searching ability of the optimization problem and to rectify the problem of solution settled at local optima. The chaos local search method is based on two well known maps such as logistic map and tent map. The chaotic local search can be described as the following logistic equation [26],

$c x_{i}^{k+1}=4 c x_{i}^{k}\left(1-c x_{i}^{k}\right) \quad i=1,2, \ldots, n$

where, $\mathrm{k}$ is the maximum number of iteration or 300

The necessary steps of the chaotic local search are as follows:

Step 1: The decision variables $x_{i}^{k}$ is converted into chaotic variables $c x_{i}^{k}$ using the equation

$$
c x_{i}^{k}=\frac{x_{i}^{k}-l b_{i}}{u b_{i}-l b_{i}} \quad i=1,2, \ldots \ldots, n
$$

where, $l b i$ and $u b i$ are the boundary value of the $x$.

Step 2: Evaluate the chaotic variables $c x_{i}^{k+1}$ for the next iteration using equation (18). 
Step 3: The chaotic variables $c x_{i}^{k+1}$ is renewed into decision variables $x_{i}^{k+1}$ using the following equation,

$$
x_{i}^{k+1}=l b_{i}+c x_{i}^{k+1}\left(u b_{i}-l b_{i}\right) \quad i=1,2, \ldots \ldots, n
$$

Step 4: Calculate the fitness of new solution with decision variables $x_{i}^{k+1}$.

Step 5: To apply greedy selection and to get the best fitness value of the problem.

\subsection{Chaotic Artificial Bee Colony (CABC) algorithm}

The ABC algorithm is successfully implemented for large scale optimization problem. Further, it requires more number of cycles for solving the optimization problem and thereby increases the computational time of the problem. To overcome this drawback, the exploitation process of $\mathrm{ABC}$ algorithm is enhanced in the proposed approach. The chaos theory is combined with $\mathrm{ABC}$ called $\mathrm{CABC}$ and which has improved the exploitation process of the searching behaviour. The computational procedure of the $\mathrm{CABC}$ algorithm as follows:Initialize the vector of the population of food sources $x_{m}(m=1,2, \ldots, C S)$, and control parameters such as limit (L), $k$ and maximum cycle number (MCN) are set, where CS (colony size) denotes as the size of the population. For optimization problem, the vector of the population of food source $\dot{x}_{m}$ holds on $n$ variables $\left(x_{m i}, i=1,2, \ldots . n\right)$ to be optimized. In these food sources used to optimize objective function of the problem.

$x_{m i}=l b_{i}+\operatorname{rand}[0,1] *\left(u b_{i}-l b_{i}\right)$

The employed bees are able to search for new food source $v_{m}$ in the dancing area containing more nectar within the neighbourhood of the food source $x_{m}$ in the memory. The new food source is determined by

$v_{m i}=x_{m i}+\operatorname{rand}[-1,1] *\left(x_{m i}-x_{k i}\right)$

where, $x_{k}$ is a randomly selected food source, $i$ is a randomly chosen parameter index. The new food source $\hat{v}_{m}$ is applied to the objective function and evaluates the corresponding fitness value. If this fitness value is improved when compared to previous one means that it will be replaced by new one. Otherwise, retain the old one and trail value is incremented by one. The onlooker bees are probabilistically to select their food sources in order to the employed bee information. The probability for each food source $m$ presented by the related employed bees can be determined as follows,

$p_{m}=\frac{f i t\left(\hat{x}_{m}\right)}{\sum_{m=1}^{S N} f i t\left(\rho_{m}\right)}$

The value of $P_{m}$ is normalized into $[0,1]$.

The probabilistically chosen of a food source $x_{m}$, and then evaluate a neighbourhood source $\tilde{v}_{m}$ using the equation (22), and its fitness value is evaluated. If this fitness value is better when compared to old one means that it will be replaced by new one. Otherwise, retain the old one and trail value is incremented by one.Further, to improve the local search ability of the problem using chaos theory equation (18), (19) and (20) to obtain the best food source from the onlooker bees. To apply the greedy selection is able to find the improved solution of the problem. The update food source in each cycle is improved which results that trial value will be set or reset to zero. Otherwise, the value of trial is incremented by one.Any one of the specific food source trial value is reached at maximum value means, the particular food source is abandoned.Using scouts bees to find the new solution of specific food source using the equation as,

$v_{m i}=l b_{i}+\operatorname{rand}[0,1] *\left(u b_{i}-l b_{i}\right)$

Finally, the improved solution stored in the memory.

\subsection{Implementation of $\mathrm{CABC}$ algorithm is the aim of optimal network reconfiguration with the location and sizing of DGs in the test systems is described as follows,}

1. Initialize the colony size (CS) and D-dimensional parameter vectors of the optimization problem

$x_{m i} ; \quad m=1,2, \ldots ., C S$

(25)The generation $m$ with CS should be constant over the entire process of the optimization. The control parameters such as food number $(\mathrm{CS} / 2)$, Limit $(\mathrm{L}), k$ and Maximum Cycle Number (MCN) are initialized.

2. To generate the random population vector using equation (21) as,

$x_{m i}=\left(x_{m 1}, x_{m 2}, \ldots, x_{m N}\right) \quad i=1,2, \ldots \ldots, N$

where, $\mathrm{N}$ is the number of control variables. In this approach, the position of the sectionals/tie switches and the location of the DGs are selected as the integer variable of the food source. The proposed CABC algorithm perfectly handles the integer variables of the problem and which is the position of food source rounding off to the nearest integer.

3. The fitness value of the objective function by using

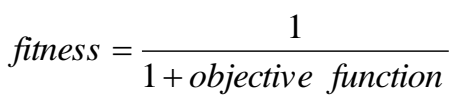

4. Run the distribution power flow and to check whether the system is radial or not. If the system is radial means the bees able to search the population in feasible region. Otherwise, the search population is infeasible region. Further, to obtain the initial best value of position of switches, location and sizing of DG units.

5. Set cycle $=1$.

6. Employed bee phase: To generate the new solution $v_{m}$ using the equation (22) and calculated them. The new food sources are used to evaluate the fitness value and satisfied the mention constraints. To update the fitness function of the problem using a greedy selection process between $v_{m}$ and $x_{m}$

7. Calculate the probability values $p_{m}$ for the solutions $x_{m}$ by using the equation (23) 
8. Onlooker bee phase: To generate the new solution $v_{m}$ in the way of disturbing food source solution $x_{m}$ on the onlooker bee phase and selected based on probability $p_{m}$ and calculate them. To update the fitness function of the problem using a greedy selection process between $v_{m}$ and $x_{m}$.

9. Chaotic local search: To improve the local search ability of bees using the chaos theory and to update the new food source. Further, to calculate the fitness value of corresponding population and to apply the greedy selection between $v_{m}$ and $x_{m}$

10. Scout bee phase: During the search process, the number of trial counter is exceeds the limit value, the solution should be abandoned. The specific food source solution should be replaced by scout bee using the equation (24) and to maintain the within limits of the population.

11. Memorize the best food source position reached so far.

12. Cycle $=$ cycle +1

13. If the number of the cycle reaches at MCN, go to step 14, otherwise go to step 5 .

14. STOP and print the result of best reconfiguration, location and sizing of DG units.The proposed method has been implemented using MATLAB 2009 running on core i3 computer with reasonable computational time.

\section{RESULTS AND DISCUSSIONS \\ 6.1.Test Systems}

The proposed approach has been implemented on the 33-node and 69-node radial distribution system consisting of base values used are $100 \mathrm{MVA}$ and $12.66 \mathrm{KV}$. The size of the DG unit is considered in a practical range of $0-1.2 \mathrm{MW}$ for both test systems. The system and load data on the 33-node system is taken from the reference [1] and the 69-node system is a reference [3]. The DG unit is delivered as a real power only and which operates at unity power factor for both test systems.

\subsection{Initialisation}

The considerations of several impact indices listed in section 2 and are used to calculate the MOPI intended at network reconfiguration, location and sizing of DG units in the test systems. In $\mathrm{CABC}$ algorithm, the control parameters are selected as, $\mathrm{CS}=50, \mathrm{MCN}=100, k=300$ and $\mathrm{L}=0.5 \times \mathrm{CS}$ $\mathrm{x} \mathrm{D}$ for both cases.

\subsubsection{3-node radial distribution system}

The results of optimal opened switches, various impact indices and MOPI for different cases of the test system are listed in table 2. The optimal result of MOPI is satisfied for all the mentioned constraints in the various cases of this approach. The implementation of the network reconfiguration topology of the system in various cases of this approach is shown in figure 2 . In this test system consists of a constant load model type and the entire node is having some committed load. Further, to run the distribution load flow for evaluating the objective of optimal opened switches in different cases of the system. During that time any one of the node voltage (having load) is exactly same in before node of the system, it will be fail the logic of radial nature of the system. For that condition, it gives much attention of the network reconfiguration and to maintain the radial nature in the case of load flow. The specific population vector is not satisfied with the radial nature of the distribution system, it will be skipped in the next generation of the algorithm.In most of the earlier report, the network reconfiguration topology is a concern for minimization of real power loss in the system. However, in this approach gives the importance of real and reactive power loss, voltage profile and stability of the system related to the various impact indices are considered and are listed in table 2.In the case I, the optimal opened switches evaluated by the $\mathrm{CABC}$ is $9,14,32,7,37$ and which results of real power loss is $139.53 \mathrm{KW}$ and reactive power loss is 102.29 KVAr. The real and reactive power loss of the system in base case condition is $202.67 \mathrm{KW}$ and $135.12 \mathrm{KVAr}$ respectively. The real power loss of this system is reduced to $31.15 \%$ compared to base case with impact of network reconfiguration. The minimum voltage stability index of case I is 0.7734 and is improved when compared to the base case of 0.6951 . The result of optimal opened switches in various existing techniques with compared to the proposed approach is listed in table 3 . The superiority of our proposed method in table 3 , the minimum voltage is 0.9378 p.u. slightly more when compared to the other methods. For the case II, the optimal reconfiguration obtained by the CABC is $13,37,35$, 6,16 and which results of real and reactive power loss are $59.39 \mathrm{KW}$ and $43.52 \mathrm{KVAr}$ respectively. The real power loss is reduced to $70.70 \%$ compared to base case and which is greatly reduced when compared to earlier methods. The minimum voltage stability index is 0.9115 and the voltage is 0.9771 p.u. listed in table 2 and 3. The optimal location and sizing of DG is listed in table 2. To select the size of DG in the distribution system should not exceed the boundary limit of DG. The impact of DG is represented that the results of case II are superior when compared to case I and is listed in table 2 and 3. 
Table 2 Results of optimal reconfiguration and various indices of different cases for 33-node system

\begin{tabular}{c|c|c|c|c|c|c|c}
\hline Optimal case & Switches opened & ILP & ILQ & VSI & CC & IVD & MOPI \\
\hline $\begin{array}{c}\text { Case 1: Network } \\
\text { reconfiguration }\end{array}$ & $9,14,32,7,37$ & 0.6885 & 0.7570 & 0.7734 & 0.8983 & 0.0622 & 0.7177 \\
\hline $\begin{array}{c}\text { Network reconfiguration with } \\
\text { location and sizing of } \\
\text { DGs (MW). }\end{array}$ & $\begin{array}{c}13,37,35,6,16 \\
\text { Case 2: } 1.12069\end{array}$ & 0.2931 & 0.3221 & 0.9115 & 0.4681 & 0.0229 & 0.3811 \\
\hline
\end{tabular}

Table 3 Comparisons of Simulation Results in different cases for 33-node system

\begin{tabular}{|c|c|c|c|c|c|}
\hline Optimal Case & Item & GA [8] & RGA [8] & HSA [8] & $\begin{array}{c}\text { CABC (Proposed } \\
\text { approach) }\end{array}$ \\
\hline \multirow{4}{*}{ Case I } & Switches opened & $33,9,34,28,36$ & $\begin{array}{c}7,9,14,32,33 \\
{[6]}\end{array}$ & $7,14,9,32,37$ & $9,14,32,7,37$ \\
\hline & $\begin{array}{l}\text { Real Power loss } \\
(\mathrm{KW})\end{array}$ & 141.60 & $139.53[6]$ & 138.06 & 139.53 \\
\hline & Power loss (\%) & 30.15 & 31.15 & 31.88 & 31.15 \\
\hline & $\begin{array}{l}\text { Minimum voltage } \\
(\text { p.u. })\end{array}$ & 0.9310 & 0.9315 & 0.9342 & 0.9378 \\
\hline \multirow{4}{*}{ Case II } & Switches opened & $7,10,28,32,34$ & $7,9,12,32,27$ & $7,14,10,32,28$ & $13,37,35,6,16$ \\
\hline & $\begin{array}{l}\text { Real Power loss } \\
\text { (KW) }\end{array}$ & 75.13 & 74.32 & 73.05 & 59.39 \\
\hline & Power loss (\%) & 62.92 & 63.33 & 63.95 & 70.70 \\
\hline & $\begin{array}{l}\text { Minimum voltage } \\
(\text { p.u. })\end{array}$ & 0.9766 & 0.9691 & 0.9700 & 0.9771 \\
\hline
\end{tabular}

The results of optimal location and sizing of DG units are listed in table 2. For case II the minimum voltage stability index of the test system is 0.9115 greater than case I which impacts of DG. The graphical representation of the voltage profile of this system is shown in figure 4 (a). In case II is a better voltage profile when compared to case I of the test system and which is evident that the value of IVD is 0.0229 less listed in table 2.

\subsubsection{9-node radial distribution system}

The evaluation of optimal opened switches, various impact indices and MOPI for different cases of this system is listed in table 4 and comparisons of existing literature in table 5. The execution of various cases in the network reconfiguration topology of this system is shown in figure 3. In this system some of the nodes are having loads (arrow) and some of the nodes are not having the load (not the arrow). In the case I, the optimal opened switches determined by the CABC is 70, $69,14,61,57$ and which effect of system the real power loss is $98.58 \mathrm{KW}$ and reactive power loss is $92.01 \mathrm{KVAr}$. The real and reactive power loss of the system in base case condition is $224.97 \mathrm{KW}$ and $102.12 \mathrm{KVAr}$ respectively. In this case, the significant reduction of real power loss of this system is $56.28 \%$ compared to base case and which is compared to other methods is listed in table 5 . The minimum voltage stability index of this case is 0.8116 and which is enhanced when compared to the base case is 0.6833 . The 
minimum voltage of this case is 0.9495 p.u. improved when compared to the existing methods consequent that better voltage profile of the system. For the case II, the optimal reconfiguration obtained by $\mathrm{CABC}$ of this system is 11,14 , $55,62,69$ and which results of real and reactive power loss are $39.59 \mathrm{KW}$ and $34.89 \mathrm{KVAr}$ respectively. The value of real power loss is lower when compared to the other method and which is shown in table 5 . The real power loss is reduced to $82.40 \%$ compared to base case and which is the better minimization of the objective in the problem when compared to the earlier methods. The minimum voltage stability index is 0.9161 and the voltage is 0.9784 p.u. listed in table 4 and 5 . The optimal location and sizing of DG is listed in table 4 and which is impacts that the voltage stability of the index is greatly improved when compared to case I.

Table 4 Results of optimal reconfiguration and various indices of different cases for 69-node system

\begin{tabular}{c|c|c|c|c|c|c|c}
\hline Optimal case & Switches opened & ILP & ILQ & VSI & CC & IVD & MOPI \\
\hline $\begin{array}{c}\text { Case1: Network } \\
\text { reconfiguration }\end{array}$ & $70,69,14,61,57$ & 0.4382 & 0.9010 & 0.8116 & 0.9466 & 0.0505 & 0.6560 \\
\hline $\begin{array}{c}\text { Case 2: } \\
\text { location and sizing of } \\
\text { DGs (MW). }\end{array}$ & $\begin{array}{c}21,14,55,62,69 \\
50 @ 1.07830 \\
61 @ 1.2\end{array}$ & 0.1760 & 0.3417 & 0.9161 & 0.5527 & 0.0216 & 0.3634 \\
\hline
\end{tabular}




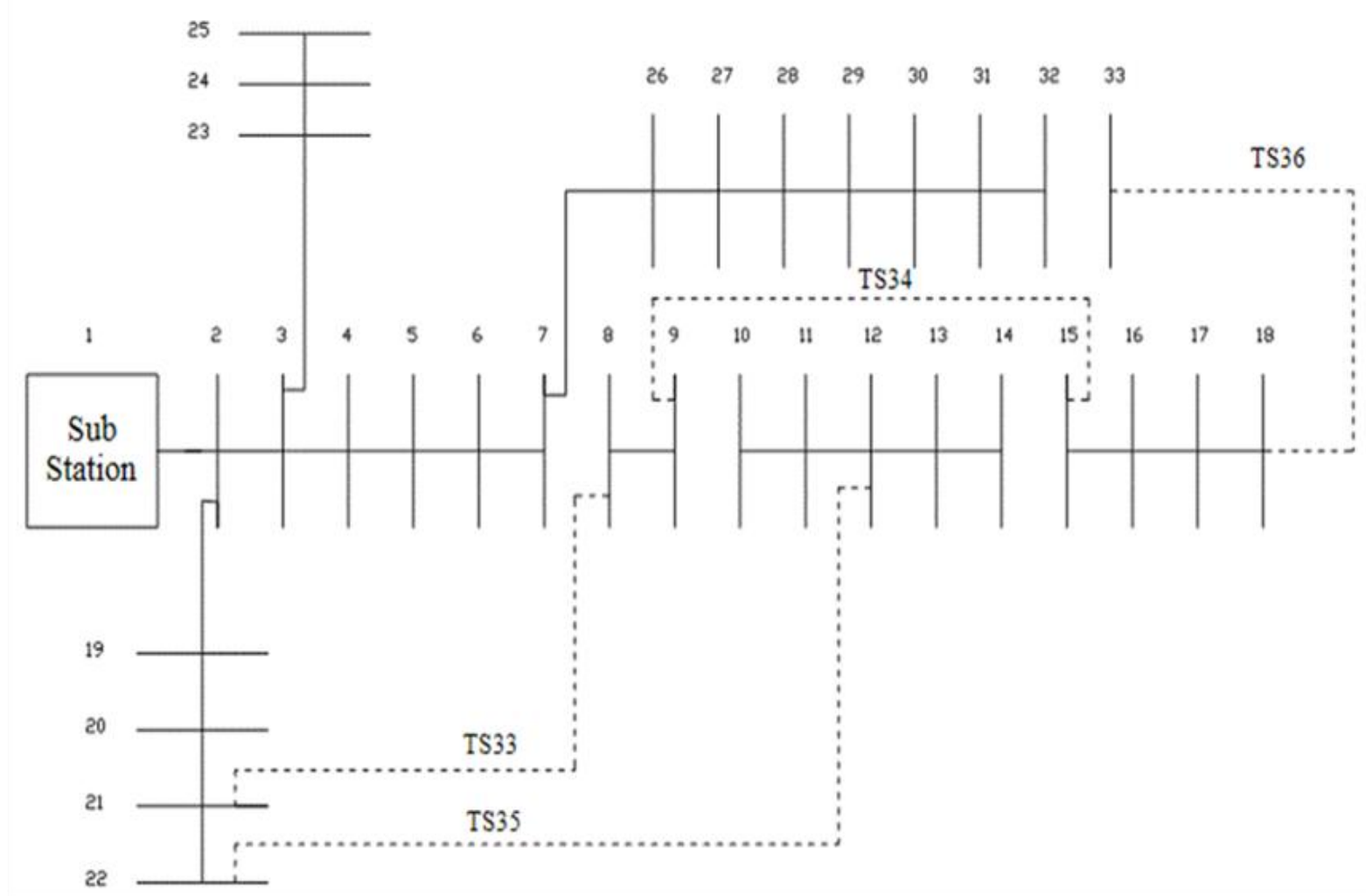

(a) Case I

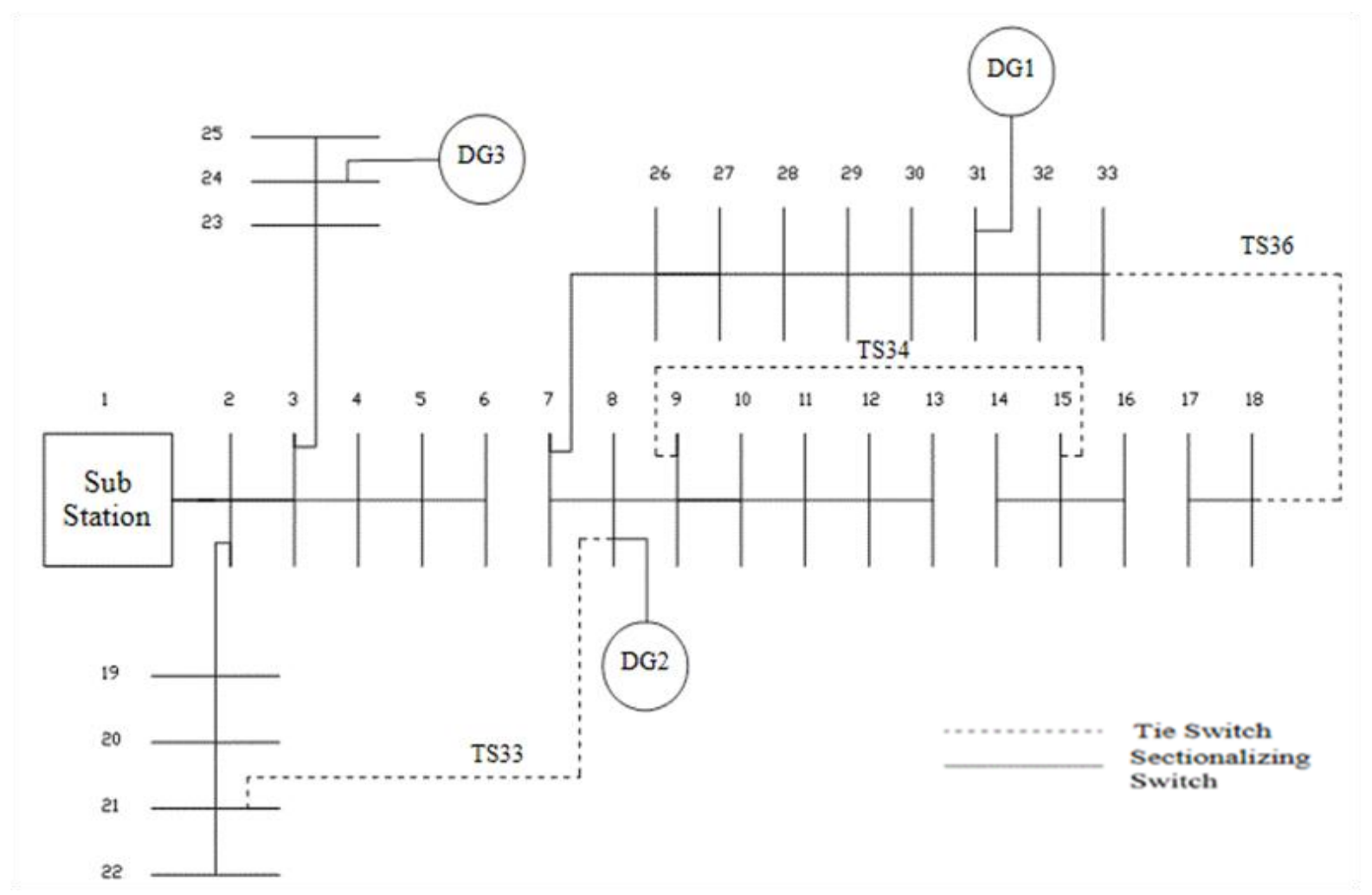

(b) Case II

Fig. 2. Network reconfiguration for various cases of 33-node system 
Table 5 Comparisons of Simulation Results in different cases for 69-node system

\begin{tabular}{|c|c|c|c|c|c|}
\hline $\begin{array}{l}\text { Optimal } \\
\text { Case }\end{array}$ & Item & GA [8] & RGA [8] & HSA [8] & $\begin{array}{c}\text { CABC } \\
\text { (Proposed } \\
\text { approach) }\end{array}$ \\
\hline \multirow{4}{*}{ Case I } & Switches opened & $69,70,14,53,61$ & $69,17,13,55,61$ & $69,18,13,56,61$ & $70,69,14,61,57$ \\
\hline & $\begin{array}{l}\text { Real Power loss } \\
(\mathrm{KW})\end{array}$ & 103.29 & 100.28 & 99.35 & 98.58 \\
\hline & Power loss $(\%)$ & 54.08 & 55.42 & 55.85 & 56.28 \\
\hline & $\begin{array}{c}\text { Minimum } \\
\text { voltage (p.u.) }\end{array}$ & 0.9411 & 0.9428 & 0.9428 & 0.9495 \\
\hline \multirow{4}{*}{ Case II } & Switches opened & $10,15,45,55,62$ & $10,16,14,55,62$ & $69,17,13,58,61$ & $11,14,55,62,69$ \\
\hline & $\begin{array}{c}\text { Real Power loss } \\
(\mathrm{KW})\end{array}$ & 46.50 & 44.23 & 40.30 & 39.59 \\
\hline & Power loss (\%) & 73.38 & 80.32 & 82.08 & 82.40 \\
\hline & $\begin{array}{c}\text { Minimum } \\
\text { voltage }(p . u .)\end{array}$ & 0.9727 & 0.9742 & 0.9736 & 0.9784 \\
\hline
\end{tabular}

The other indices are also reasonably reduced with impacts of network reconfiguration with DGs as shown in table 4. The graphical representation of the voltage profile of this system is shown in figure 4 (b). The $\mathrm{CC}$ index is reasonably reduced by the impact of the proposed approach in different cases are listed in table 2 and 4 for the test systems. The presence of DG in the system, it is possible of supplying power near to loads with the effects of current flows in some branches of the network are increased in the current carrying capacity. 


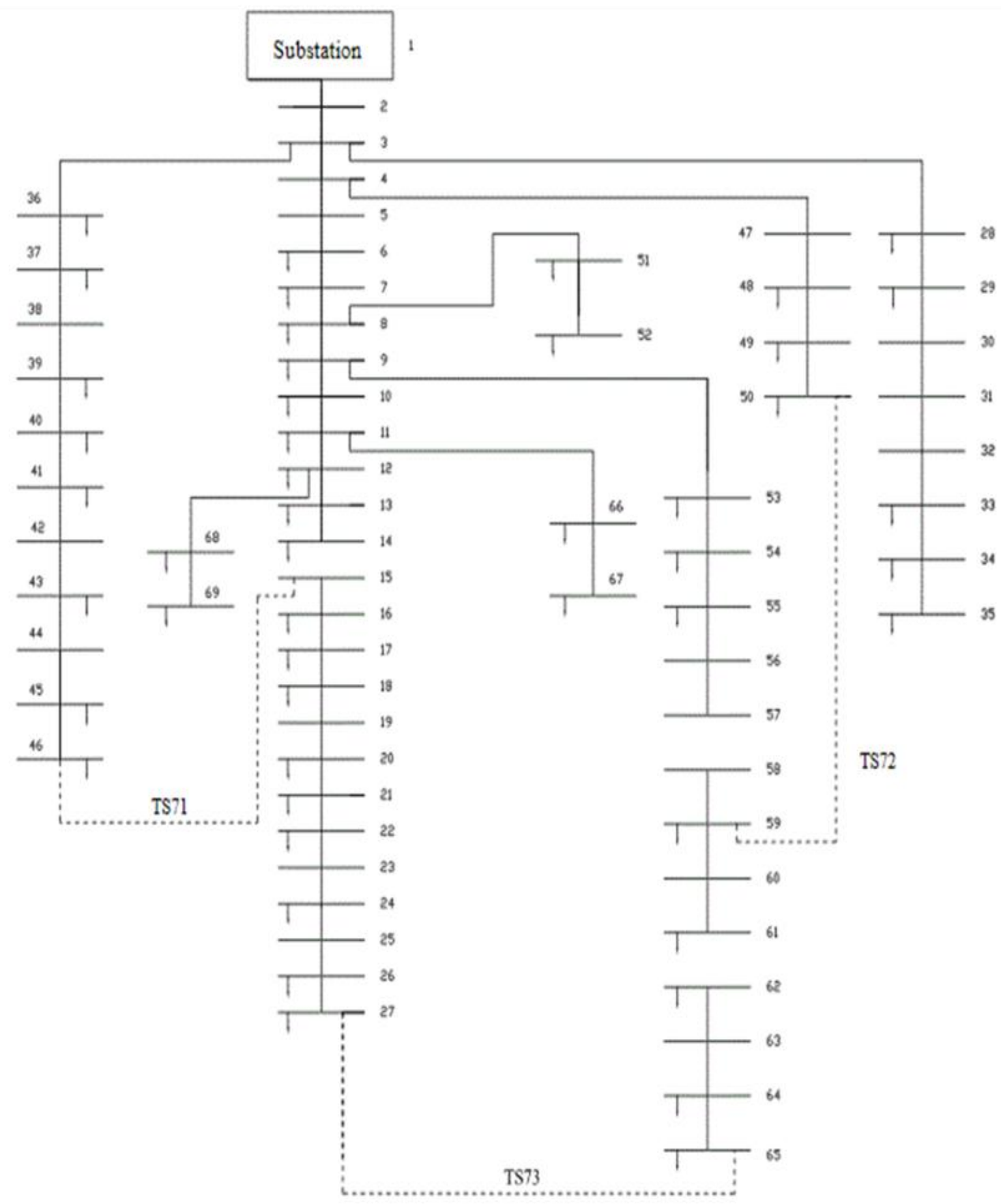

(a) Case I

The presented value of CC index is lesser than one is indicated that current flows are within limit and the system operates in a safe manner. The convergence characteristic curves of 33-node and 69-node system by using CABC algorithm is represented in figure $5 \& 6$. It will be clearly seen that the performance of the CABC algorithm and the corresponding optimal values of the problem is evaluated up to the maximum number of iterations (cycle). 


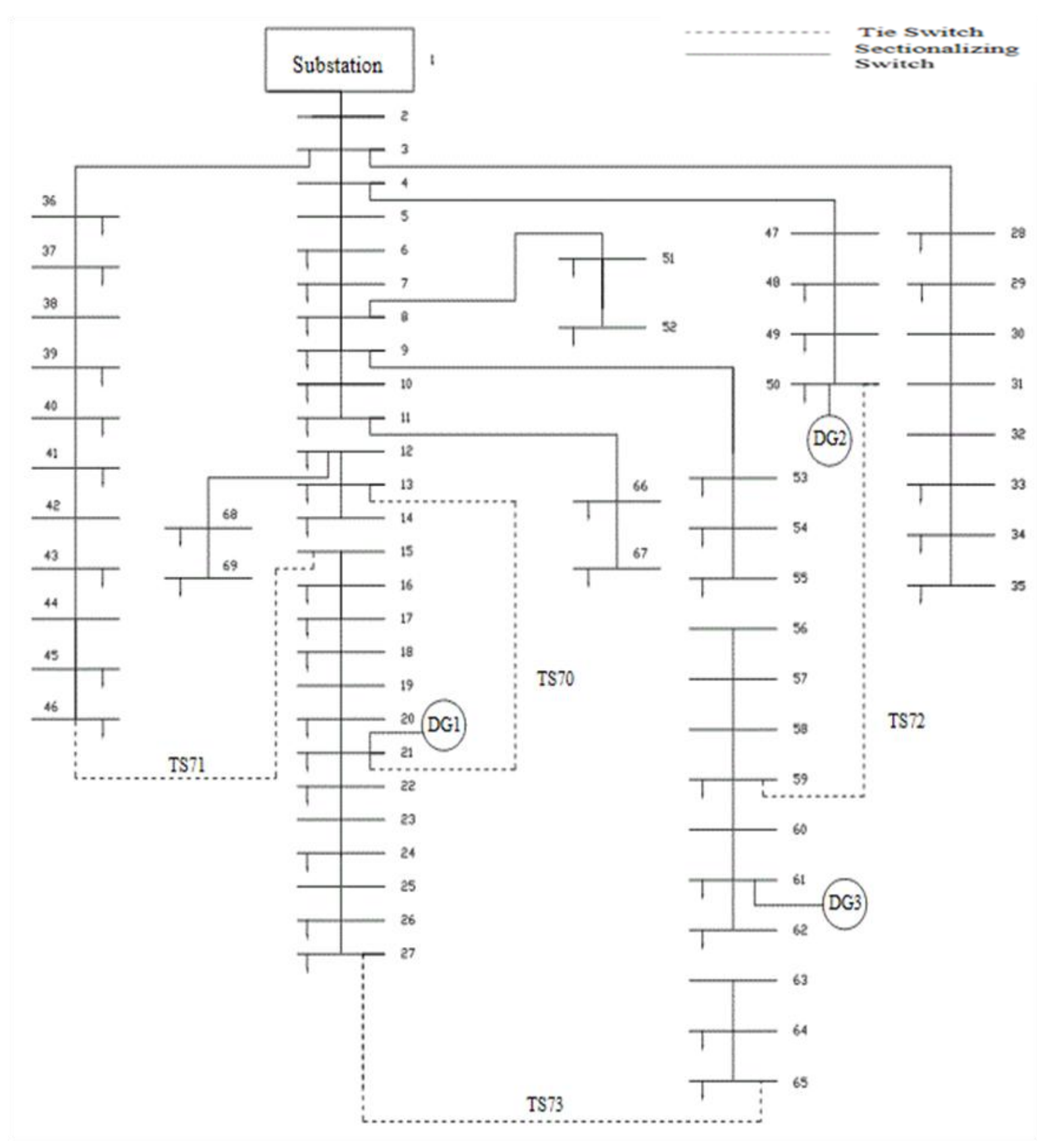

(b) Case II

Fig. 3. Network reconfiguration for various cases of 69-node system 


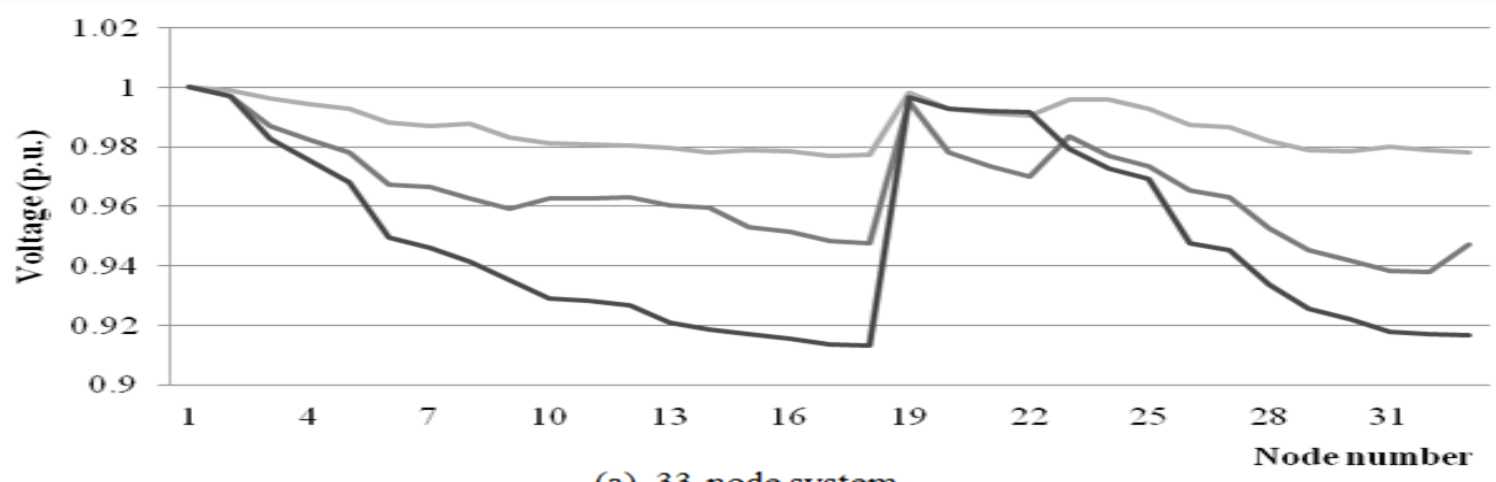

(a). 33-node system

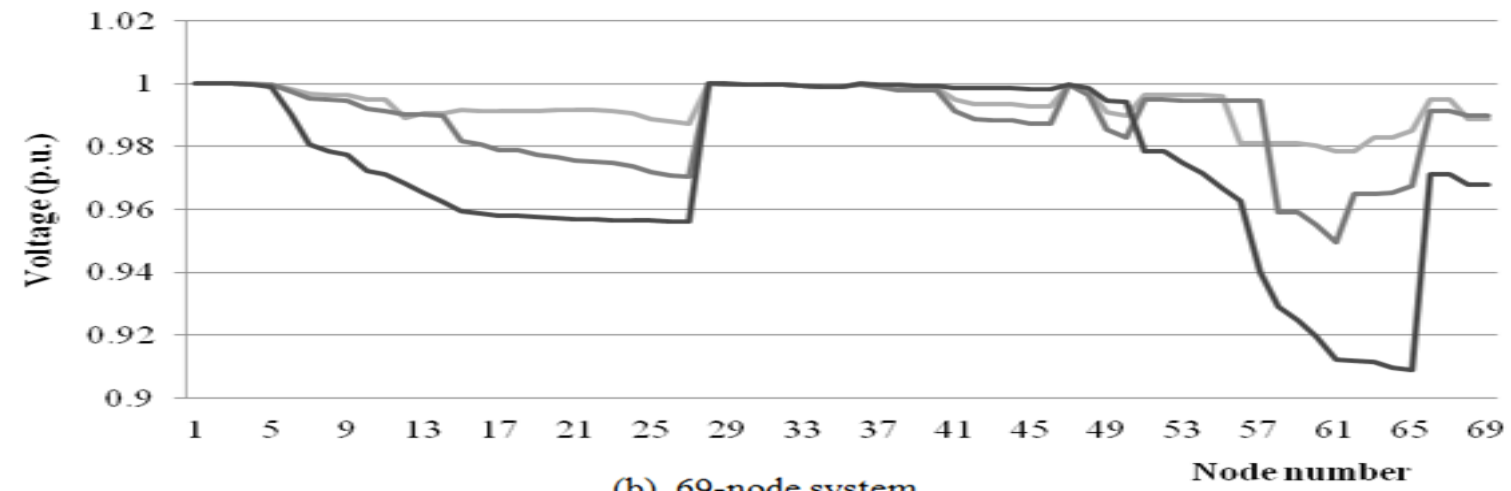

(b). 69-node system

Network Reconfiguration with DG Network Reconfiguration

Base case

Fig. 4. Voltage profile of system with various cases

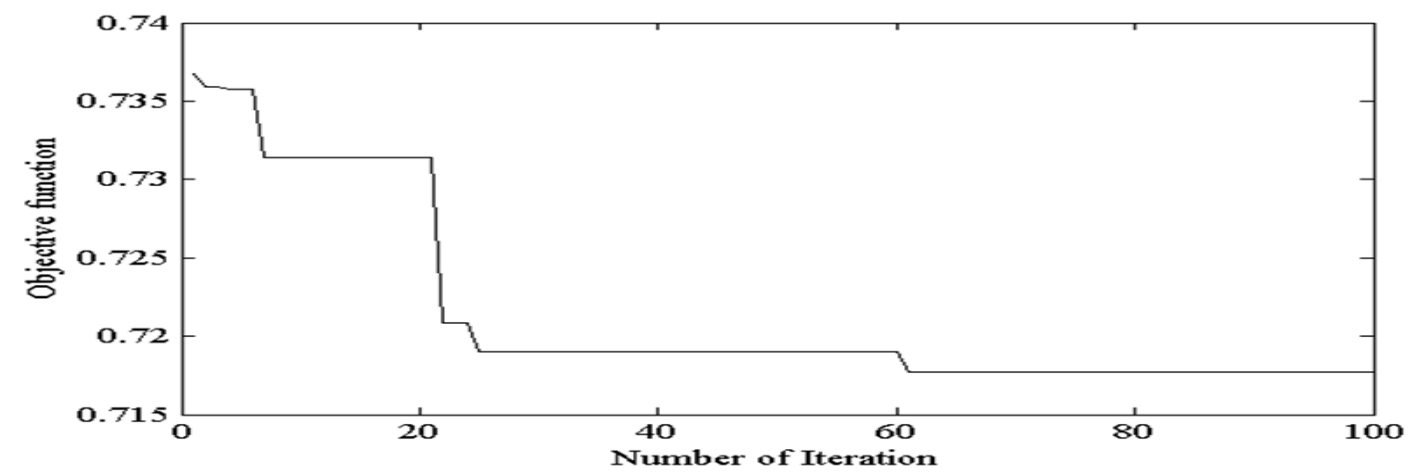

(a) Case I

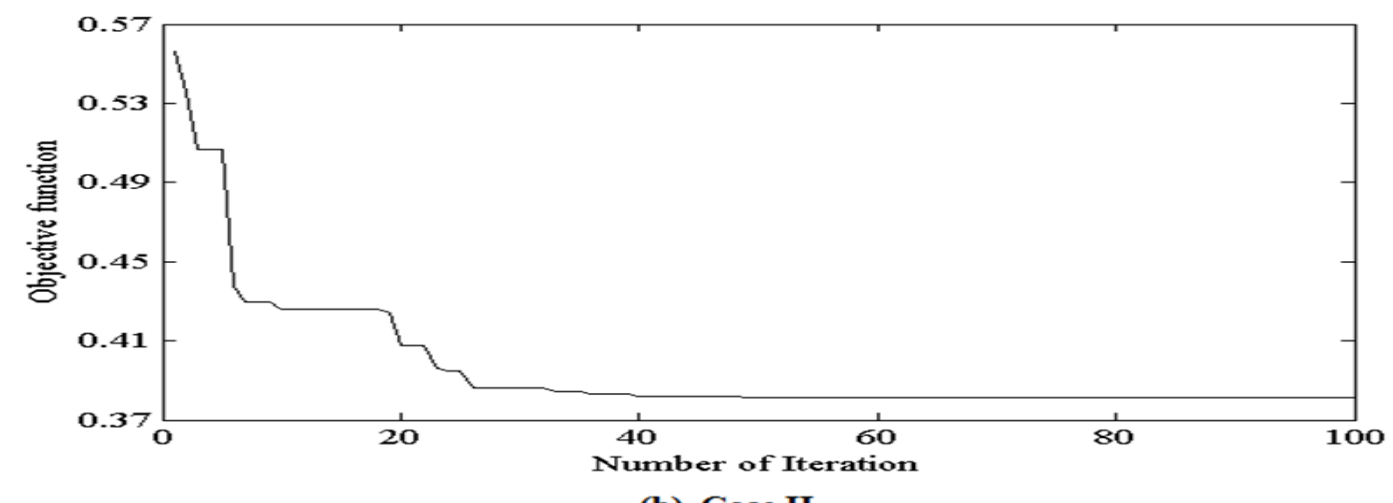

(b) Case II

Fig. 5. Convergence characteristic curve for various cases of 33-node system 


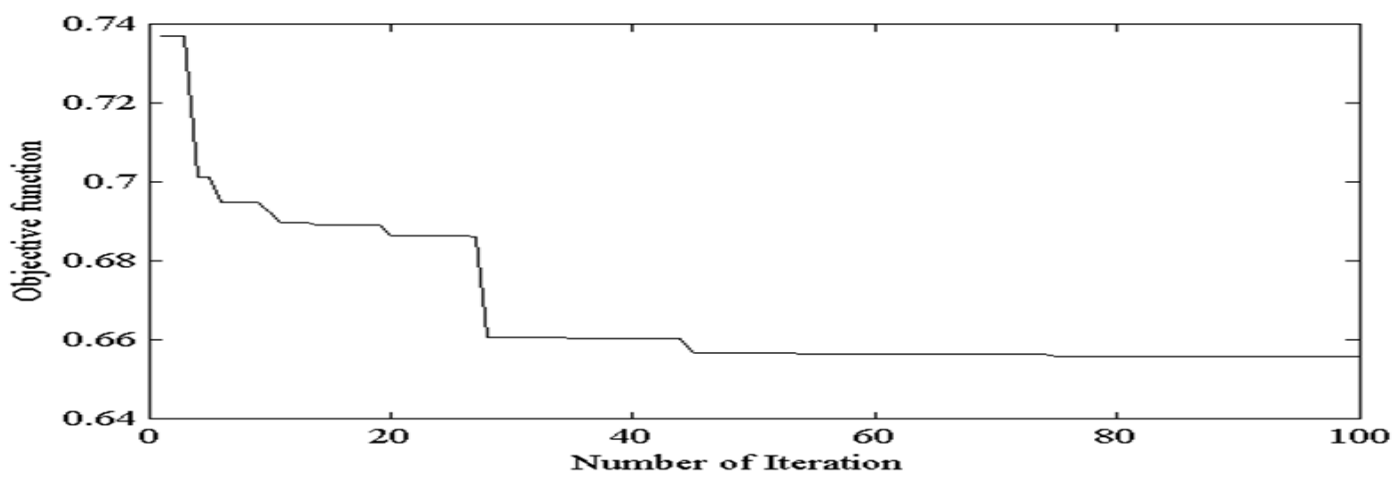

(a) Case I

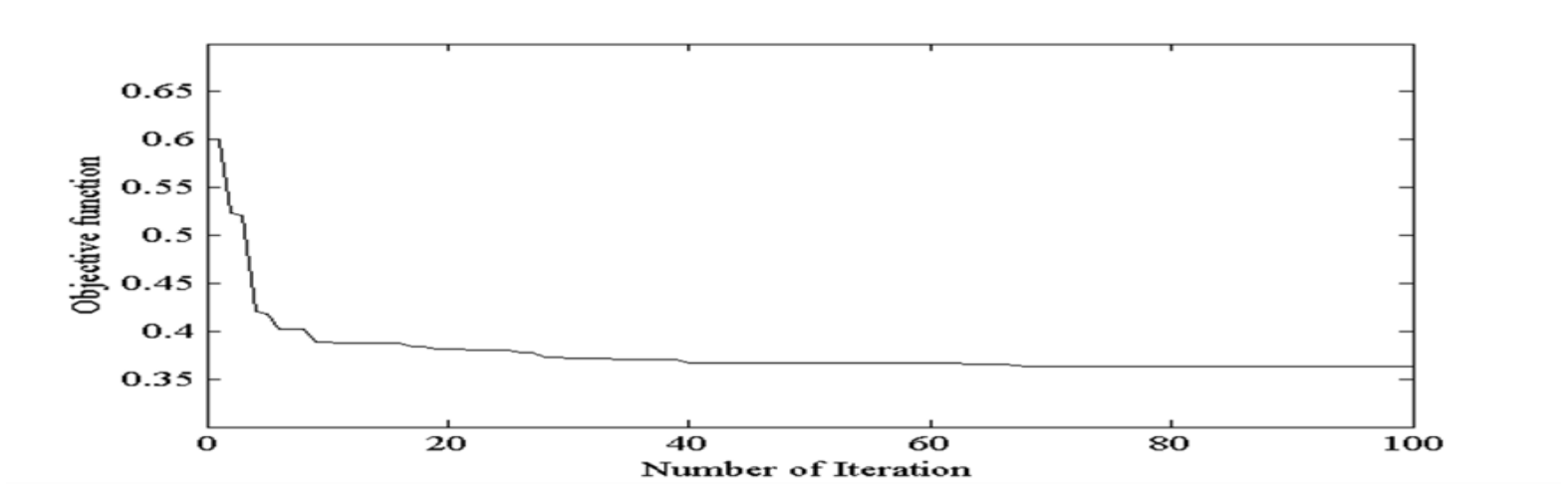

(b) Case II

Fig. 6. Convergence characteristic curve for various cases of 69-node system

\section{7. . CONCLUSION}

In this paper, the voltage stability of the radial distribution systems is improved by network reconfiguration topology with the additional of optimal location and sizing of the DGs. The MOPI is formulated as impact indices of various technical issues and is normalized between zero and one. The final results of these indices are normalized consequent that better improvement of such issues and is verified with tables. In this method, the real power loss of the test systems is significantly reduced for various cases when compared to the existing methods. The voltage profile and stability of the system are enhanced for gradually applied the different cases of the problem. The current capacity index gives information about for upgrades of the feeder. As per the result, the CABC algorithm based proposed method is superior when compared to the existing method.

\section{ACKNOWLEDGMENT}

The author gratefully acknowledges the authorities of Srirangam Polytechnic College for the extensive facilities provided to carry out this research work.

\section{REFERENCES}

[1] M. E. Baran and F. F. Wu, "Network Reconfiguration in Distribution Systems for Loss Reduction and Load Balancing", IEEE Trans. Power Del., vol. 4, no. 2, pp. 1401-1407, Apr. 1989.

[2] B. Venkatesh, R. Ranjan, and H. B. Gooi, "Optimal Reconfiguration of Radial Distribution Systems to Maximize Loadability", IEEE Trans. Power Sys.,vol. 19, no. 1, pp. 260-266, Feb. 2004.

[3] J. S. Savier and D. Das, "Impact of Network Reconfiguration on Loss Allocation of Radial
Distribution Systems", IEEE Trans. on Power Del., vol. 22, no. 4, pp. 2473-2480, Oct. 2007.

[4] P. V. V. Rama Rao and S. Sivanagaraju, "Radial Distribution Network Reconfiguration for Loss Reduction and Load Balancing using Plant Growth Simulation Algorithm", Intern. J. Elect. Engg. and Inform., vol. 2, no. 4, pp. 266-277, Nov. 2010.

[5] J. Olamaei, T. Niknam, and G. Gharehpetian, "Application of particle swarm optimization for distribution feeder reconfiguration considering distributed generators", Applied Mathematics and Computation 201, pp. 575-586, 2008.

[6] J. Z. Zhu, "Optimal reconfiguration of electrical distribution network using the refined genetic algorithm", Elect. Power Syst. Res., vol. 62, pp. 37-42, Jan. 2002.

[7] R. S. Rao, S. V. L. Narasimham, M. R. Raju, and A. S. Rao, "Optimal Network Reconfiguration of Large-Scale Distribution System Using Harmony Search Algorithm", IEEE Trans. Power Syst., vol. 26, no. 3, pp. 1080-1088, Aug. 2011.

[8] R. S. Rao, K. Ravindra, K. Satish, and S. V. L. Narasimham, "Power Loss Minimization in Distribution System Using Network Reconfiguration in the Presence of Distributed Generation", IEEE Trans. Power Syst., vol. 28, no. 1, pp. 317-325, Feb. 2013.

[9] G. K. V. Raju and P. R. Bijwe, "An Efficient Algorithm for Minimum Loss Reconfiguration of Distribution System Based on Sensitivity and Heuristics", IEEE Trans. Power Syst., vol. 23, no. 3, pp. 1280-1287, Aug. 2008. 
[10] N. C. Sahoo and K. Prasad, "A fuzzy genetic approach for network reconfiguration to enhance voltage stability in radial distribution systems", Energy Conv. and Managt., 47, pp. 3288-3306, Mar. 2006.

[11] C. F. Chang, "Reconfiguration and Capacitor Placement for Loss Reduction of Distribution systems by Ant Colony Search Algorithm", IEEE Trans. Power Syst., vol. 23, no. 4, pp. 1747-1755, Nov. 2008.

[12] M. Sedighizadeh, M. Dakhem, M. Sarvi and H. H. Kordkheili, "Optimal reconfiguration and capacitor placement for power loss reduction of distribution system using improved binary particle swarm optimization", Int J Energy Environ Eng., 5:73, pp. 1-11, 2014.

[13] G. Pepermans, J. Driesen, D. Haeseldonckx, R. Belmans, and W. D'haeseleer, "Distributed generation: definition, benefits and issues", Energy Policy 33, pp. 787-798, 2005.

[14] D. Das, "Optimal placement of capacitors in radial distribution system using a Fuzzy-GA method", Electrical Power and Energy Syst., 30, pp. 361-367, 2008.

[15] D. Singh, R. K. Misra, and D. Singh, "Effect of Load models in distributed generation planning", IEEE Trans. Power Syst., vol. 22, no. 4, pp. 2204-2212, Nov. 2007.

[16] D. Singh, D. Singh, and K. S. Verma, "Multiobjective optimization for DG planning with load models", IEEE Trans. Power Syst., vol. 24, no. 1, pp. 427-436, Feb. 2009.

[17] N. Mohandas, R. Balamurugan, and L. Lakshminarasimman, "Optimal location and sizing of real power DG units to improve the voltage stability in the distribution system using $\mathrm{ABC}$ algorithm united with chaos", Electrical Power and Energy Systems 66, pp. 41$52,2015$.
[18] M. Chakravorty, and D. Das, "Voltage stability analysis of radial distribution networks", Electrical Power and Energy Systems 23, pp. 129-135, 2001.

[19] D. Thukaram, H.M.W. Banda, and J. Jerome, "A robust three-phase power flow algorithm for radial distribution systems", Electric Power Systems Research 50, pp. 227 236, 1999.

[20] S. Ghosh and D. Das, "Method for load-flow solution of radial distribution networks", IEE Proc. Gener. Transm. Distrib., vol. 146, no. 6, pp. 641-648, Nov. 1999.

[21] D. Karaboga, and B. Basturk, "A powerful and efficient algorithm for numerical function optimization: artificial bee colony (ABC) algorithm", Journal of Global Optimization 39, pp. 459-471, Apr. 2007.

[22] B. Akay and D. Karaboga, "Artificial bee colony algorithm for large-scale problems and engineering design optimization”, J Intell Manuf 23, pp. 1001-1014, Mar. 2012.

[23] D. Karaboga, and B. Akay, "A comparative study of Artificial Bee Colony algorithm", Applied Maths. and Compt., 214, pp. 108-132, 2009.

[24] B. Akay and D. Karaboga, "A modified Artificial Bee Colony algorithm for real-parameter optimization", Inform. Sciences, vol. 192, 1, pp. 120-142, June 2012.

[25] Q. Pan, M. F. Tasgetiren, P. N. Suganthan, and T. J. Chua, "A discrete artificial bee colony algorithm for the lot-streaming flow shop scheduling problem", Information Sciences 181, pp. 2455-2468, 2011.

[26] B. Liu, L. Wang, Y. Jin, F. Tang and D. Huang, "Improved particle swarm optimization combined with chaos", chaos solitons and fractals 25, pp. 1261-1271, 2005.

[27] R. T. Marler and J. S. Arora, "The weighted sum method for multi-objective optimization: new insights", Struct Multidisc Optim., pp. 1-10, Dec. 2009. 